\title{
Doenças Crônicas Não Transmissíveis na Revista Ciência \& Saúde Coletiva: um estudo bibliométrico
}

\author{
Noncommunicable diseases in the Journal Ciência \& Saúde \\ Coletiva: a bibliometric study
}

Deborah Carvalho Malta (https://orcid.org/0000-0002-8214-5734) ${ }^{1}$

Alanna Gomes da Silva (https://orcid.org/0000-0003-2587-5658) ${ }^{2}$

Laís Santos de Magalhães Cardoso (https://orcid.org/0000-0002-1114-5470) ${ }^{1}$

Fabiana Martins Dias de Andrade (https://orcid.org/0000-0001-8277-6061) ${ }^{1}$

Ana Carolina Micheletti Gomide Nogueira de Sá (https://orcid.org/0000-0002-0122-2727) ${ }^{1}$

Elton Junio Sady Prates (https://orcid.org/0000-0002-5049-186X) ${ }^{1}$

Francielle Thalita Almeida Alves (https://orcid.org/0000-0001-8886-8514) ${ }^{1}$

Gesner Francisco Xavier Junior (https://orcid.org/0000-0001-5190-3523) ${ }^{2}$

${ }^{1}$ Escola de Enfermagem, Universidade Federal de Minas Gerais (UFMG). Av. Professor Alfredo Balena 190, Santa Efigênia. 30130 100 Belo Horizonte MG Brasil.dcmalta@uol.com.br ${ }^{2}$ Biblioteca José Baeta Vianna, UFMG. Belo Horizonte MG Brasil.

\begin{abstract}
Noncommunicable Diseases are an important public health issue in Brazil and worldwide. This study aimed to analyze the scientific production published by the "Journal Ciencia \& Saúde Coletiva" in order to shed light on its contributions for the dissemination of scientific knowledge and the debate regarding noncommunicable diseases. A bibliometric study on the publications from 1996 to 2019 related to noncommunicable diseases was carried out. A total of 458 documents that met the eligibility criteria were selected. An increasing trend in the number of publications per year was found; at some points, it coincided with political and institutional milestones in Brazil. Quantitative research papers stood out, as did studies on risk and protective factors. Public educational and research institutions led the publishing and the financing of the studies. Most authors were female. The journal has been reflecting the magnitude of the theme and its prioritization on the public agenda by promoting the debate and providing a scientific dissemination of content related to noncommunicable diseases.
\end{abstract}

Key words Noncommunicable diseases, Public Health, Risk factors, Scientific communication and diffusion, Bibliometrics
Resumo As Doenças Crônicas Não Transmissíveis configuram importante problema de saúde pública no Brasil e no mundo. Visando conferir visibilidade às contribuições da revista Ciência \& Saúde Coletiva para a divulgação do conhecimento científico e o debate das questões relacionadas às Doenças Crônicas Não Transmissíveis, o presente estudo teve por objetivo analisar a produção científica publicada pela revista. Realizou-se um estudo bibliométrico das publicações veiculadas no período de 1996 a 2019, referentes a essas doenças. Com base em critérios de elegibilidade, selecionou-se um total de 458 publicações. Observou-se tendência temporal de aumento das publicações, coincidente com o estabelecimento de marcos politico-institucionais no país. Destacaram-se os artigos quantitativos e os estudos sobre fatores de risco e proteção. Evidenciou-se a liderança das instituições públicas de ensino e pesquisa na produção científica sobre o tema e, também, no financiamento público dos estudos. Os autores que mais publicaram são majoritariamente do sexo feminino. A revista vem refletindo a magnitude e a prioridade da temática na agenda pública ao promover o debate e oportunizar a divulgação científica sobre as doenças crônicas.

Palavras-chave Doenças crônicas não transmissíveis, Saúde pública, Fatores de risco, Comunicação e divulgação científica, Bibliometria 


\section{Introdução}

As Doenças Crônicas Não Transmissíveis (DCNT) constituem importante problema de saúde pública, haja vista serem a principal causa de morte no mundo, além de ocasionarem mortalidade prematura, incapacidades, perda da qualidade de vida, sobrecarga no sistema de saúde $\mathrm{e}^{1,2} \mathrm{e}$ de contribuírem para o aumento dos gastos com assistência médica e previdência social ${ }^{3,4}$.

$\mathrm{O}$ aumento da morbimortalidade por essas doenças está relacionado aos feitos da transição epidemiológica, demográfica e nutricional ${ }^{5-7}$ e também ao crescimento de fatores de risco modificáveis como consumo de tabaco, uso nocivo de bebida alcoólica, inatividade física e alimentação inadequada $^{8}$. A esses elementos somam-se, ainda, os efeitos das crises econômicas e das medidas de austeridade ${ }^{9,10}$, e de outros determinantes sociais, em particular a pobreza, na ocorrência e na distribuição das DCNT e seus fatores de ris$\mathrm{Co}^{11,12}$. Como consequência, observam-se piores indicadores de saúde na população socialmente mais vulnerável e maiores prevalências de fatores de risco das DCNT em indivíduos com baixa escolaridade e renda ${ }^{13-15}$.

As DCNT, seus fatores de risco e de proteção, aspectos relativos ao tratamento e a ações estratégicas para seu enfrentamento, figuram entre as temáticas abordadas nas publicações da revista Ciência \& Saúde Coletiva (C\&SC), que espelha a relevância do tema nos cenários nacional e global. A revista, editada pela Associação Brasileira de Saúde Coletiva (Abrasco), foi criada em 1996 com o objetivo de divulgar resultados de pesquisas em saúde de alta qualidade para a comunidade científica e para a sociedade em geral, no Brasil e no exterior. Trata-se de importante veículo nacional de comunicação científica de discussões, debates, pesquisas e de exposição de novas ideias no campo da Saúde Coletiva, além de estar indexado nas principais bases de dados em saúde.

Considerando-se a relevância das DCNT nos contextos brasileiro e global e na perspectiva de lançar luz às importantes contribuições da C\&SC nesse campo temático, o presente estudo teve por objetivo analisar a produção científica referente às DCNT publicada na revista, que está completando 25 anos de existência.

\section{Métodos}

Trata-se de estudo exploratório e descritivo que consistiu na análise bibliométrica do acervo da
C\&SC publicado no período de 1996 a 2019. A bibliometria corresponde a um conjunto de métodos de pesquisa que alia a abordagem quantitativa, a estatística e as técnicas de visualização de dados ${ }^{16}$ aplicadas à análise e avaliação da produção bibliográfica, com a intenção de medir índices de produção e de disseminação do conhecimento científico ${ }^{17}$.

A C\&SC está indexada em diversas bases de dados. Para recuperar as publicações de interesse, utilizou-se a base de dados Medical Literature Analysis and Retrieval System Online (Medline), via United States National Library of Medicine National Institutes of Health (PubMed), bem como a Scientific Electronic Library Online (SciELO). A PubMed foi utilizada como buscador por ser extremamente robusta e confiável e por ter permitido a elaboração de uma estratégia de busca com todos os termos necessários para representar o universo pesquisado. Ressalva-se, porém, que não foi possível recuperar, via PubMed, o conteúdo da C\&SC publicado entre 1996 e 2006, pois o mesmo não se encontra indexado na Medline. Sendo assim, fez-se necessário segmentar o processo de mapeamento da produção veiculada na C\&SC em três etapas:

Primeira etapa: levantamento de informações gerais sobre o que foi publicado na revista no período de 1996 a 2019 para contextualização. Foram extraídas da SciELO Analytics, tendo em vista que o conteúdo integral da revista está indexado nesta plataforma.

Segunda etapa (recuperação manual): recuperação das publicações referentes às DCNT. Realizou-se busca "manual", ou seja, feita a varredura em todo o material publicado entre os anos de 1996 e 2006, por meio da SciELO, para mapear os manuscritos elegíveis não indexados na Medline.

Terceira etapa (recuperação automatizada): recuperação das publicações referentes às DCNT. Para identificar os manuscritos elegíveis publicados entre 2007 e 2019, construiu-se uma estratégia de busca no PubMed, a qual foi realizada em fevereiro de 2020, a partir de descritores extraídos do Medical Subject Headings (MeSH), acrescidos dos respectivos sinônimos e palavras-chave, que não estavam contempladas na lista dos sinônimos. Foram utilizados termos relacionados aos quatro grandes grupos de DCNT e seus fatores risco modificáveis, que foram associados por meio dos operadores “OR” e "AND”. Considerou-se os seguintes MeSH: Noncommunicable Diseases, Cardiovascular Diseases, Diabetes Mellitus, Respiratory Tract Diseases, Neoplasms, Hypertension, Healthy Lifestyle, Health Behavior, Body 
Weight, Obesity, Body Mass Index, Body Weights and Measures, Weight Gain, Overweight, Healthy Diet, Sedentary Behavior, Motor Activity, Tobacco, Tobacco Use Disorder, Tobacco Use, Tobacco Smoking, Smokers, Smoking, Cigarette Smoking, Tobacco Products, Alcoholism, Alcoholic Beverages, Alcoholics, Dyslipidemias, Binge Drinking, Risk Factors.

Como critério de inclusão, considerou-se todos os tipos de publicação veiculados na revista (editorial, artigos temáticos, artigos de temas livres, artigos de revisão, opinião, resenhas, debates e cartas) que apresentassem como enfoque: a) os quatro principais grupos de doenças priorizadas pela Organização Mundial da Saúde no "Plano de Ação Global para a Prevenção e Controle das DCNT 2013-2020" e no "Plano de Ações Estratégicas para o Enfrentamento das DCNT no Brasil, 2011-2022"18 (circulatórias, câncer, respiratórias crônicas e diabetes); b) os fatores de risco modificáveis (tabaco, álcool, inatividade física, alimentação inadequada) e os metabólicos (obesidade, hipertensão arterial, glicemia alterada); c) políticas, programas e ações referentes às DCNT, vigilância, promoção, prevenção, diagnóstico, cuidado e tratamento. Os critérios de exclusão compreenderam publicações que não apresentassem como objetivo principal as DCNT nas dimensões de abordagem referidas anteriormente.

Orientada pelos critérios de elegibilidade, a seleção das publicações foi feita, em ambas as etapas (busca manual e por meio do PubMed), com base na leitura do título, do resumo e das palavras-chave, no caso de documentos do tipo artigo e do texto completo, quando se tratava de documentos do tipo editorial, opinião, debate, resenha e carta. Essa leitura foi realizada por pares, as divergências de seleção foram discutidas e resolvidas por consenso entre as partes e os motivos da exclusão das publicações foram registrados.

Os dados de interesse das publicações selecionadas foram extraídos para uma planilha Microsoft Office Excel (Microsoft ${ }^{\circledR}$ 2016). Considerouse as seguintes variáveis: número identificador da publicação, sendo o PubMed Unique Identifier (PMID) para as indexadas na Medline e o Document Object Identifier (DOI) para as não indexadas nesta base; ano de publicação; idioma; seção da publicação; autoria (quantidade de autores por artigo e nome dos autores); vinculação institucional dos autores; palavras-chave; financiamento; categorias temáticas e link de acesso às publicações na plataforma SciELO.

As categorias temáticas foram pautadas pelas dimensões de abordagem das DCNT menciona- das no critério de inclusão. Assim sendo, cada publicação foi classificada em uma dessas quatro categorias: 1) morbimortalidade, 2) fatores de risco e de proteção, 3 ) estratégias terapêuticas e de cuidado, e 4) políticas, programas e ações. $\mathrm{Na}$ categoria de morbimortalidade foram incluídos artigos relacionados com os quatro grandes grupos de DCNT, diagnóstico e mortalidade por essas doenças. Para fatores de risco e de proteção entraram publicações que tinham como objeto os fatores de risco (comportamentais, estilos de vida, biológicos, metabólicos, ambientais, desigualdades sociais, econômicas e demográficas) e os fatores protetivos para as DCNT (estilos de vida saudável, promoção e prevenção da saúde). As estratégias terapêuticas e de cuidado agruparam estudos com o foco em tratamento das DCNT, pesquisas farmacológicas, estratégias de cuidado integral às DCNT e autocuidado. A categoria políticas, programas e ações contemplou publicações que abordaram políticas, ações programáticas para DCNT, vigilância e monitoramento.

Realizou-se análise descritiva dos dados a partir do levantamento das frequências absolutas e relativas para cada variável. Em adição, empregou-se o método de regressão linear simples para verificar a ocorrência de tendência linear do número de publicações no período de 1996 a 2019. Considerou-se como variável explicativa o ano de publicação dos manuscritos na C\&SC e como variável resposta a quantidade de publicações (frequências absolutas). As variações temporais foram interpretadas como estatisticamente significativas quando o coeficiente de regressão do modelo se mostrou diferente de zero para um valor de $\mathrm{p}$ menor ou igual a 0,05. As análises estatísticas foram realizadas no software Microsoft Office Excel (Microsoft ${ }^{\circledast}$ 2016).

Nuvens de palavras-chave e de vinculação institucional dos autores das publicações foram elaboradas com auxílio da ferramenta de visualização de dados online Infogram (https://infogram. com/). A "nuvem de palavras", ou "nuvem de texto", é uma proposta visual de apresentação de dados linguísticos que mostra a frequência com que os termos aparecem e propicia identificar a importância de determinada palavra em relação ao número total de palavras ${ }^{19}$. As palavras-chave contempladas nos artigos selecionados foram agrupadas de acordo com seus sinônimos, segundo o vocabulário do "Descritores em Ciências da Saúde (DeCS)", assim como termos no plural e no singular e vocábulos em idiomas diferentes, mas com o mesmo significado. Além disso, para 
melhor visualização dos termos nas figuras, optou-se por estabelecer um ponto de corte com base na distribuição de frequências absolutas, ordenada da maior para a menor frequência, de modo que a nuvem de palavras-chave apresentou 29 termos de um total de 625, enquanto que a nuvem de vinculações institucionais dos autores conferiu visibilidade às 50 mais recorrentes, de um total de 251 instituições.

\section{Resultados}

Entre 1996 e 2019, a C\&SC publicou 247 números e 5.871 documentos, dos quais 4.860 foram artigos de pesquisa. A revista teve periodicidade semestral entre 1996 e 2001, quadrimestral de 2002 a 2006, bimestral entre 2007 e 2010 e, a partir de 2011, passou a ser mensal, com a publicação anual de doze números.

Destaca-se o lançamento do primeiro número temático dedicado às DCNT em 2004. Observa-se que as doenças crônicas ou os temas relacionados (pesquisas nacionais, vigilância, fatores de risco como obesidade e hipertensão e relativos ao comportamento alimentar e ao estilo de vida) também foram assuntos de interesse explicitados nos títulos e em tópicos contemplados nos editoriais de números temáticos, a exemplo dos publicados nas edições de: 2004 (número 4), 2010 (suplemento 2), 2011 (número 1), 2013 (número 2), 2014 (números 1 e 6), 2015 (números 3, 4 e 8), 2016 (números 1, 2 e 4), 2017 (número 2), 2018 (número 12) e 2019 (número 7).

Na recuperação manual foram identificados, inicialmente, um total de 794 publicações e após exclusões restaram 25 . Na recuperação automatizada, a estratégia de busca recuperou 979 documentos, dos quais selecionou-se 433 com base nos critérios de elegibilidade. Ao final obteve-se um total de 458 artigos incluídos.

A Tabela 1 apresenta o panorama das publicações segundo período, seção da revista, número de autores, idioma, categoria temática e financiamento. Ao considerar o recorte temporal de 25 anos da revista C\&SC, o primeiro manuscrito que atendeu ao critério de elegibilidade deste estudo foi publicado em 2002. Observou-se um grande salto no quantitativo de publicações sobre DCNT nos anos seguintes.

Os artigos correspondem ao tipo de publicação majoritária, perfazendo um total de 440 documentos $(96,0 \%)$, dos quais a maioria utilizou metodologia quantitativa $(72,1 \%)$. Houve predomínio de publicações com 3 e 4 autores (39,7\%)
Tabela 1. Publicações da Revista Ciência \& Saúde Coletiva sobre DCNT, segundo características gerais, 2002 a 2019.

\begin{tabular}{|c|c|c|}
\hline Características & $\mathbf{n}$ & $\%$ \\
\hline \multicolumn{3}{|l|}{ Total de publicações $(\mathrm{N}=458)$} \\
\hline \multicolumn{3}{|l|}{ Período das publicações } \\
\hline 2002 a 2005 & 22 & 4,8 \\
\hline 2006 a 2011 & 107 & 23,4 \\
\hline 2012 a 2015 & 149 & 32,5 \\
\hline 2016 a 2019 & 180 & 39,3 \\
\hline \multicolumn{3}{|l|}{ Seção da publicação } \\
\hline Artigos & 440 & 96,0 \\
\hline Quantitativo & 330 & 72,1 \\
\hline Revisão & 54 & 11,8 \\
\hline Qualitativo & 52 & 11,4 \\
\hline Misto & 3 & 0,7 \\
\hline Reflexão & 1 & 0,2 \\
\hline Debate & 7 & 1,5 \\
\hline Carta & 4 & 0,9 \\
\hline Editorial & 3 & 0,7 \\
\hline Opinião & 3 & 0,7 \\
\hline Resenha & 1 & 0,2 \\
\hline \multicolumn{3}{|l|}{ Número de autores } \\
\hline 1 e 2 & 94 & 20,5 \\
\hline 3 e 4 & 182 & 39,7 \\
\hline 5 e 6 & 130 & 28,4 \\
\hline 7 a 12 & 52 & 11,4 \\
\hline \multicolumn{3}{|c|}{ Vinculação institucional dos autores $(\mathrm{N}=1912)^{\mathrm{a}}$} \\
\hline Instituição pública & 1646 & 86,0 \\
\hline Instituição privada & 266 & 13,9 \\
\hline Não se aplica & 1 & 0,1 \\
\hline \multicolumn{3}{|l|}{ Idioma } \\
\hline Português & 279 & 60,9 \\
\hline Português e inglês & 108 & 23,6 \\
\hline Inglês & 66 & 14,4 \\
\hline Espanhol & 4 & 0,9 \\
\hline Espanhol e inglês & 1 & 0,2 \\
\hline \multicolumn{3}{|l|}{ Categoria Temática } \\
\hline Fatores de risco e de proteção & 257 & 56,1 \\
\hline Morbimortalidade & 98 & 21,4 \\
\hline Políticas, programas e ações & 54 & 11,8 \\
\hline Estratégias terapêuticas e cuidado & 49 & 10,7 \\
\hline \multicolumn{3}{|l|}{ Financiamento ${ }^{\mathrm{b}}$} \\
\hline $\operatorname{Sim}$ & 99 & 21,6 \\
\hline $\begin{array}{l}\text { Agências nacionais de fomento à } \\
\text { pesquisa }\end{array}$ & 80 & 53,0 \\
\hline $\begin{array}{l}\text { Fundações estaduais de amparo à } \\
\text { pesquisa }\end{array}$ & 40 & 26,5 \\
\hline Órgãos governamentais & 20 & 13,2 \\
\hline Outros & 11 & 7,3 \\
\hline Não declarado & 344 & 75,1 \\
\hline Não se aplica & 15 & 3,3 \\
\hline
\end{tabular}

Fonte: Elaborado pelos autores.

${ }^{\mathrm{a} A s} 458$ publicações em questão totalizaram uma indicação de 1912 vinculações institucionais (considerando repetições). ${ }^{\mathrm{b}} \mathrm{A}$ soma das frequências absolutas dos agentes financiadores excede $o$ total de artigos que declararam $\operatorname{sim}(\mathrm{n}=151$ versus $\mathrm{n}$ =99), pois, em alguns casos, um mesmo artigo declarou mais de uma fonte de financiamento. 
e as instituições públicas representaram a vinculação institucional mais prevalente $(86,0 \%)$. A maior parte das publicações foi escrita e publicada em português $(60,9 \%)$, embora uma parcela notável tenha sido redigida e publicada em português e inglês ou somente em inglês $(38,0 \%)$. "Fatores de Risco e de Proteção" foi a categoria temática mais frequente $(56,1 \%)$, seguida por "Morbimortalidade" $(21,4 \%)$. Das publicações analisadas, pequeno percentual declarou apoio financeiro $(26,1 \%)$, e, deste, as agências nacionais de fomento à pesquisa figuraram como o maior agente financiador (53,0\%), seguidas pelas fundações estaduais de amparo à pesquisa $(26,5 \%)$ (Tabela 1$)$.

Um total de 1.529 pesquisadores distintos, ou seja, desconsiderando-se as repetições, foi responsável pela produção das 458 publicações analisadas. Desses, 27 contribuíram em 4 ou mais publicações, seja como primeiro autor ou como coautor e, em sua maioria, são do sexo feminino $(70,4 \%)$. O ponto de corte de 4 ou mais publicações foi definido para melhor apresentação dos dados, considerando o grande quantitativo de autores que participaram de 3 ou menos publicações ( $\mathrm{n}=1502$; dados não mostrados). Os cinco autores que mais publicaram foram: Malta DC; Barros MBA; Cotta RMM; Barreto SM; Christofaro DGD. No tocante à vinculação institucional destes pesquisadores, destacam-se as instituições públicas, sendo as universidades federais as mais frequentes (Tabela 2).

A Figura 1 apresenta o quantitativo anual de publicações segundo categorias temáticas (A), e a linha do tempo do total anual de publicações de acordo com marcos institucionais do campo das DCNT (B). Como já mencionado anteriormente, as primeiras publicações sobre DCNT foram verificadas na C\&SC em 2002. Observa-se predomínio das publicações na temática "morbimortalidade" em 2002 e 2004. A partir de 2008, a temática "fatores de risco e de proteção" das DCNT se tornou a mais frequente, sobretudo nos anos de 2015, 2018 e 2019, contabilizando, somente nesta categoria, 30, 34 e 44 publicações, respectivamente (Figura $1 \mathrm{~A}$ ).

O lançamento do primeiro número temático da C\&SC dedicado às DCNT ocorreu em 2004. $\mathrm{Na}$ sequência cronológica, destacam-se os picos em 2008, 2011, 2013, 2015, 2018 e 2019, os quais podem ser relacionados a eventos e ações nacionais e internacionais no âmbito das doenças crônicas. Em 2008, foram lançadas as "Diretrizes e Recomendações para o Cuidado Integral de DCNT" e ocorreu a inclusão de informações sobre morbidade e alguns fatores de risco na
Pesquisa Nacional por Amostra de Domicílios (PNAD), como parte da iniciativa Global Adult Tobacco Survey (GATS). Em 2011, no âmbito nacional, destacaram-se a publicação do "Plano de Ações Estratégicas para o Enfrentamento das Doenças Crônicas Não Transmissíveis (DCNT) no Brasil, 2011-2022", e o lançamento do "Programa Academia da Saúde", e, no âmbito internacional, a realização da Reunião de Alto Nível sobre DCNT, na Assembleia Geral da Organização das Nações Unidas (ONU), em Nova Iorque. Em 2013, houve o lançamento do "Plano de Ação Global para Prevenção e Controle de DCNT”, da Organização Mundial da Saúde (OMS), enquanto que, no contexto nacional, estava sendo criado o Departamento de Doenças e Agravos Não Transmissíveis e Promoção da Saúde (DANTPS), bem como a realização do primeiro inquérito da Pesquisa Nacional de Saúde (PNS). No ano de 2018, ressalta-se o lançamento da iniciativa SAFER, pela OMS, na Terceira Conferência de Alto Nível das Nações Unidas sobre a Prevenção e Controle das Doenças Não Transmissíveis, com o intuito de fomentar e fortalecer ações de redução do consumo nocivo do álcool (Figura 1B).

Entre 2002 e 2019 pode-se afirmar que há uma tendência de aumento significativo ( $\mathrm{p}<$ 0,05) no número de publicações sobre DCNT, representando uma variação média de 0,0072 ao ano, com $\mathrm{R}^{2}$ ajustado de 0,62 (dados não mostrados).

A Figura 2 apresenta as principais palavraschave presentes nas publicações analisadas. Do total de 458 publicações foram identificadas 625 palavras-chave (o somatório das frequências corresponde a um total de 1.775 palavras-chave). A nuvem de palavras mostra um recorte das 29 palavras-chave mais frequentes, entre as quais destacam-se obesidade $(n=61)$, câncer $(n=61)$, adolescentes $(n=60)$, comportamento alimen$\operatorname{tar}(\mathrm{n}=55)$, tabagismo $(\mathrm{n}=52)$, fatores de risco e proteção $(\mathrm{n}=47)$, atividade física $(\mathrm{n}=45)$, hipertensão $(\mathrm{n}=45)$, idosos $(\mathrm{n}=45)$ e doenças crônicas não transmissíveis ( $\mathrm{n}=41$ ) (Figura 2).

A Figura 3 apresenta as vinculações institucionais mais frequentes dos autores das publicações analisadas. As publicações totalizaram 251 vinculações institucionais distintas, cujo somatório das frequências perfaz o total 1.912 citações. A nuvem de palavras expõe um recorte das 50 instituições mais frequentes, das quais as que mais se destacaram foram a Universidade Federal de Minas Gerais (UFMG) $(\mathrm{n}=132)$, a Fundação Oswaldo Cruz (Fiocruz) $(\mathrm{n}=129)$ e a Universidade de São Paulo (USP) $(\mathrm{n}=118)$ (Figura 3). 
Tabela 2. Autores que contribuíram em 4 ou mais publicações da Ciência \& Saúde Coletiva sobre DCNT, segundo posição de autoria, volume total de publicações e afiliação institucional, 2002 a 2019.

\begin{tabular}{|c|c|c|c|c|}
\hline Autores & $\begin{array}{c}11^{\circ} \\
\text { autor }\end{array}$ & Coautor & Total & Vinculação institucional \\
\hline \multirow[t]{2}{*}{ Deborah Carvalho Malta } & 11 & 10 & 21 & Ministério da Saúde \\
\hline & & & & Universidade Federal de Minas Gerais \\
\hline Marilisa Berti de Azevedo Barros & 2 & 5 & 7 & Universidade Estadual de Campinas \\
\hline Rosângela Minardi Mitre Cotta & 1 & 6 & 7 & Universidade Federal de Viçosa \\
\hline Sandhi Maria Barreto & 1 & 6 & 7 & Universidade Federal de Minas Gerais \\
\hline \multirow[t]{4}{*}{ Diego Giulliano Destro Christofaro } & 1 & 5 & 6 & Universidade Estadual de Londrina \\
\hline & & & & Universidade Estadual Paulista Júlio de \\
\hline & & & & Mesquita Filho \\
\hline & & & & Universidade Federal Rural de Pernambuco \\
\hline Efigênia Ferreira e Ferreira & 0 & 6 & 6 & Universidade Federal de Minas Gerais \\
\hline \multirow[t]{2}{*}{ Luana Giatti } & 0 & 6 & 6 & Universidade Federal de Minas Gerais \\
\hline & & & & Universidade Federal de Ouro Preto \\
\hline \multirow[t]{3}{*}{ Rômulo Araújo Fernandes } & 2 & 4 & 6 & Universidade do Oeste Paulista \\
\hline & & & & Universidade Estadual Paulista Júlio de \\
\hline & & & & Mesquita Filho \\
\hline Diego Augusto Santos Silva & 1 & 4 & 5 & Universidade Federal de Santa Catarina \\
\hline Gustavo Velasquez-Melendez & 0 & 5 & 5 & Universidade Federal de Minas Gerais \\
\hline Patrícia Maria Zarzar & 0 & 5 & 5 & Universidade Federal de Minas Gerais \\
\hline Pedro Curi Hallal & 1 & 4 & 5 & Universidade Federal de Pelotas \\
\hline Andréia Queiroz Ribeiro & 0 & 4 & 4 & Universidade Federal de Viçosa \\
\hline Bruna Celestino Schneider & 1 & 3 & 4 & Universidade Federal de Pelotas \\
\hline \multirow[t]{2}{*}{ Danielle Cristina Guimarães da Silva } & 2 & 2 & 4 & Universidade Federal de Viçosa \\
\hline & & & & Universidade Federal do Oeste da Bahia \\
\hline Eliana Zandonade & 0 & 4 & 4 & Universidade Federal do Espírito Santo \\
\hline Erika Aparecida Silveira & 2 & 2 & 4 & Universidade Federal de Goiás \\
\hline Jamile Sanches Codogno & 0 & 4 & 4 & $\begin{array}{l}\text { Universidade Estadual Paulista Júlio de } \\
\text { Mesquita Filho }\end{array}$ \\
\hline \multirow[t]{2}{*}{ Lenildo de Moura } & 0 & 4 & 4 & Ministério da Saúde \\
\hline & & & & Organização Pan-Americana da Saúde \\
\hline Maria Helena Costa Amorim & 0 & 4 & 4 & Universidade Federal do Espírito Santo \\
\hline \multirow[t]{2}{*}{ Maria Teresa Anselmo Olinto } & 0 & 4 & 4 & Universidade do Vale do Rio dos Sinos \\
\hline & & & & $\begin{array}{l}\text { Universidade Federal de Ciências da Saúde } \\
\text { de Porto Alegre }\end{array}$ \\
\hline Marise Fagundes Silveira & 0 & 4 & 4 & Universidade Estadual de Montes Claros \\
\hline Mônica Rodrigues Campos & 0 & 4 & 4 & Fundação Oswaldo Cruz \\
\hline \multirow[t]{2}{*}{ Otaliba Libânio de Morais Neto } & 0 & 4 & 4 & Ministério da Saúde \\
\hline & & & & Universidade Federal de Goiás \\
\hline Romeu Gomes & 2 & 2 & 4 & Fundação Oswaldo Cruz \\
\hline Rosely Sichieri & 0 & 4 & 4 & Universidade do Estado do Rio de Janeiro \\
\hline Vanessa Alves Ferreira & 3 & 1 & 4 & $\begin{array}{l}\text { Universidade Federal dos Vales do } \\
\text { Jequitinhonha e Mucuri }\end{array}$ \\
\hline
\end{tabular}

Fonte: Elaborado pelos autores.

\section{Discussão}

O estudo aponta um crescimento contínuo da produção científica relacionada às DCNT na revista C\&SC desde 2002, sendo 2011, 2015, 2018 e
2019 os anos de publicações mais elevadas sobre a temática. Dos artigos analisados, a maioria teve abordagem quantitativa, em cerca de dois quintos das publicações o número de autores variou de 3 a 4 por artigo, e mais da metade foi publica- 


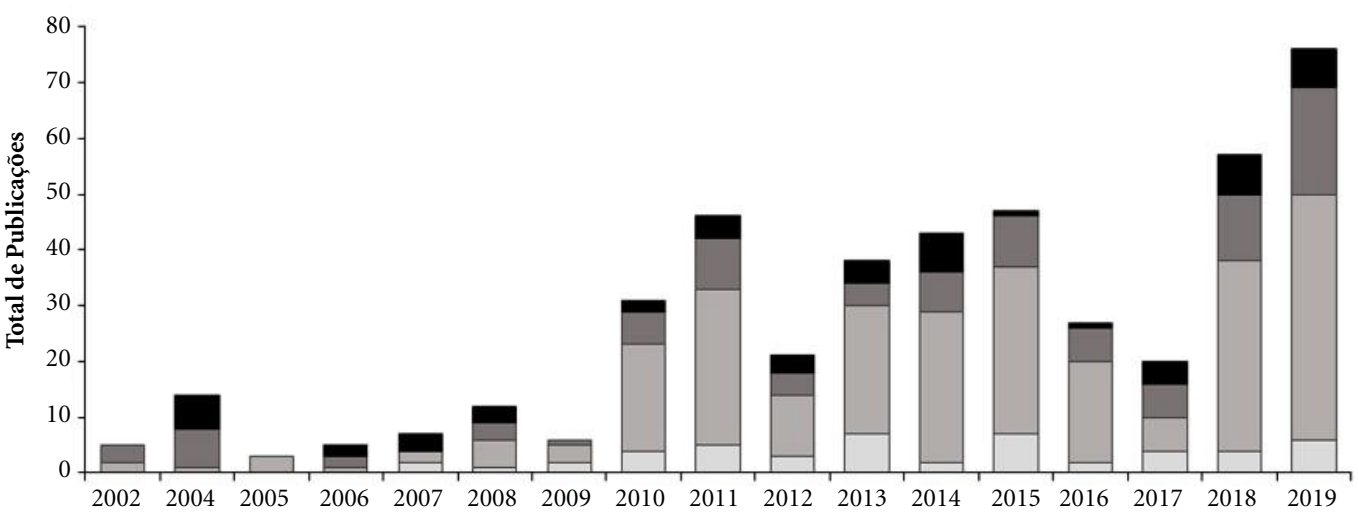

Ano

Estratégias terapêuticas e cuidado
Fatores de risco e de proteção
Morbimortalidade
Políticas, programas e ações

(B)

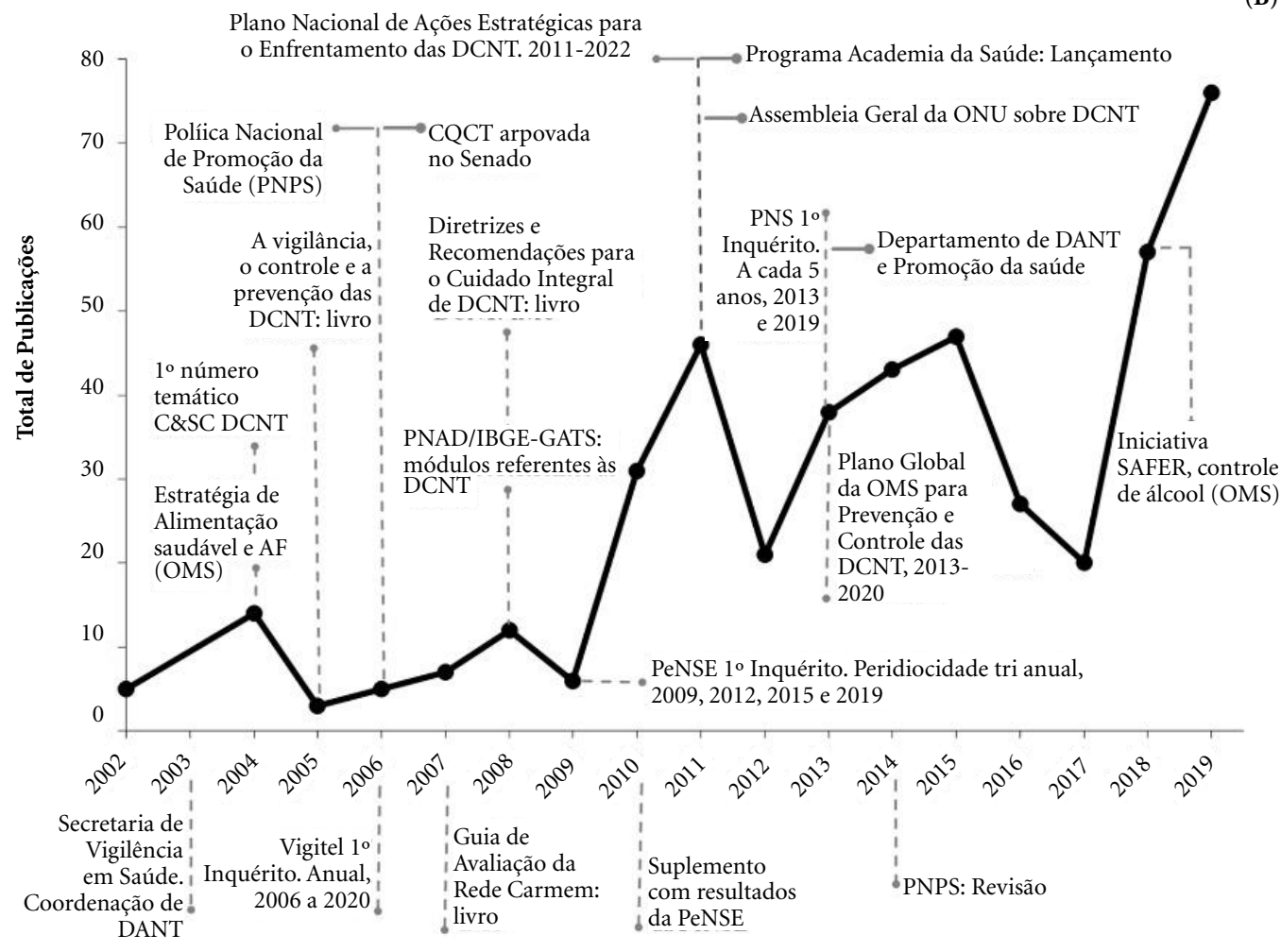

Figura 1. Número anual de publicações segundo categorias temáticas (1A) e número anual de publicações segundo marcos institucionais sobre as DCNT (1B), 2002 a 2019.

Fonte: Elaborado pelos autores. 


\section{Atenção Primária à Saúde \\ Atividade física \\ Tabagismo Diabetes \\ Adolescentes Mortalidade \\ Hipertensão Câncer Idosos \\ Álcool Obesidade \\ Comportamento alimentar}

Fatores de risco e de proteção

Doenças crônicas não transmissíveis

Figura 2. Palavras-chave mais frequentes nas publicações da Ciência \& Saúde Coletiva sobre DCNT, 2002 a 2019.

Fonte: Elaborado pelos autores.

Figura 3. Instituições mais frequentes vinculadas aos autores das publicações da Ciência \& Saúde Coletiva sobre DCNT, 2002 a 2019.

Fonte: Elaborado pelos autores.

da em português. A principal categoria temática correspondeu aos "fatores de risco e de proteção". $\mathrm{O}$ apoio financeiro foi declarado em apenas um quinto dos estudos, mas, entre os que declararam, houve predominância de agentes públicos. As instituições públicas foram, também, as que mais publicaram na revista, sendo a UFMG, a Fiocruz e a USP as principais vinculações institucionais dos autores. Pesquisadores do sexo feminino foram maioria entre os que figuraram como autores em 4 ou mais publicações. Os maiores volumes de publicações foram coincidentes com o estabelecimento de marcos político-institucionais em âmbito nacional e internacional. As palavras- chave mencionadas com maior frequência foram obesidade, câncer, adolescentes, comportamento alimentar, tabagismo e fatores de risco e proteção.

$\mathrm{O}$ aumento das publicações sobre DNCT e seus fatores de risco e proteção na C\&SC ao longo dos anos pode estar relacionado com a priorização do tema nas agendas nacional e internacional. Nessa perspectiva, é importante ressaltar a inserção das DCNT na agenda global em 2000, quando a OMS aprovou a Resolução 53.17 da Assembleia Mundial da Saúde, com objetivo de apoiar os Estados Membros em seus esforços para reduzir o número de morbidades, incapacidades e mortalidade prematura relacionadas às doenças não transmissíveis. Para isso, orientou a organização da vigilância dessas doenças, a criação de políticas públicas de promoção da saúde e de prevenção, bem como a inovação em cuidados de saúde e gestão ${ }^{20}$. No cenário nacional, a instituição de marcos governamentais e científicos foi fundamental para consolidação da temática na agenda interna, a exemplo da criação da Secretaria de Vigilância em Saúde (SVS), no Ministério da Saúde (MS), em 2003, da realização de estudos longitudinais e dos inquéritos populacionais de abrangência nacional (domiciliares, telefônicos e escolares) e municipal (ISACamp, ISA-SP e outros $)^{2}$. No organograma da SVS instituiu-se a Coordenação Geral de Vigilância de Doenças e Agravos Não Transmissíveis (CGDANT), cuja missão consistiu em consolidar o sistema de vigilância e de monitoramento das doenças e agravos não transmissíveis, implantando, então, os grandes inquéritos nacionais ${ }^{2,21,22}$.

Esses marcos governamentais também coincidem com as primeiras publicações da C\&SC, que aconteceram apenas em 2002, cerca de seis anos após o lançamento da revista. Na primeira publicação, Furquin et al. ${ }^{23}$ analisaram os dados da Pesquisa Nacional de Amostra de Domicílios (PNAD/98) do IBGE. O artigo discutiu os efeitos do envelhecimento da população brasileira, o aumento das DCNT e a forte demanda na procura dos serviços de saúde. Em outro estudo, Leite et al. ${ }^{24}$ compararam as informações sobre as prevalências de doenças crônicas obtidas pelo suplemento saúde da PNAD/98 e as estimadas pelo estudo Carga de Doença no Brasil, enquanto que Iwakami e Sono $\hat{e}^{25}$ compararam informações sobre saúde da população brasileira, baseada em dados da PNAD/98, e inquéritos norte-americanos.

Em 2004, a C\&SC publicou o seu primeiro número temático dedicado às DCNT. No editorial, Lessa ${ }^{26}$ debate a importância do tema e o atraso da sua entrada na agenda do país, as de- 
sigualdades nas estimativas de mortalidade por DCNT, afetando mais a população de baixa renda, bem como a escassez de dados sobre morbidade. Lessa ${ }^{27}$ destacou, também, a importância da aprovação, pela OMS, da estratégia Global de alimentação saudável, atividade física e saúde, bem como as iniciativas da Secretaria de Vigilância em Saúde, do Ministério da Saúde brasileiro, na implementação desta agenda afirmativa. O número temático incluiu análise de Achutti e Azambuja ${ }^{28}$ e os debatedores Loureiro ${ }^{29}$, Lotufo ${ }^{30}$ e Teixeira ${ }^{31}$, que discutiram as repercussões das DCNT e o aumento da pressão sobre a seguridade social, bem como as consequências para a sociedade. Ainda neste número, Almeida-Filho ${ }^{32}$ revisou os marcos referenciais e os modelos teóricos de determinação social das DCNT; Lima-Costa et al..$^{33}$ analisaram dados de inquérito domiciliar realizado em Belo Horizonte e apresentam as diferenças de estilos de vida e de uso de serviços preventivos de saúde entre adultos filiados ou não a plano privado de saúde, apontando piores indicadores entre aqueles que não têm planos de saúde. Laurenti et $\mathrm{al}^{34}$ analisaram os resultados de mortalidade de DCNT oriundos do Sistema de Informação de Mortalidade (SIM), enquanto Toscano ${ }^{35}$ descreveu sobre a experiência de rastreamento populacional de diabetes. O número ainda apresentou o relato governamental de Solla ${ }^{36}$, que abordou as políticas do SUS para a promoção da saúde e a prevenção das DCNT, assim como os desafios na capacitação de profissionais. Um segundo relato descreveu a primeira "Oficina de Vigilância de Doenças e Agravos Não Transmissíveis", realizada em 2004, no Congresso de Epidemiologia da Associação Brasileira de Saúde Coletiva (Abrasco), com a presença de especialistas e da CGDANT, marcando o início da organização da vigilância de DCNT no País ${ }^{37}$.

Nos anos seguintes houve publicação de novos números temáticos, com destaque para a análise dos resultados da primeira Pesquisa Nacional de Saúde do Escolar (PeNSE - 2010), abordando a saúde dos adolescentes ${ }^{38}$, a exposição ao tabagismo $^{39}$, o comportamento alimentar ${ }^{40}$, a imagem corporal $^{41}$ e a prática de atividade física ${ }^{42}$. Mais suplementos sobre alimentação e nutrição, obesidade, estilos de vida, resultados da PNS (2015), consumo de álcool e outros vieram na sequência.

No tocante ao número de autores das publicações analisadas neste estudo, predominou a pesquisa em grupo em detrimento da individual. Outros autores apontam fatos convergentes e indicam ser essa uma característica de estudos no campo das ciências da saúde ${ }^{43-45}$. A pesquisa em grupo é uma expectativa em pesquisas financiadas, haja vista que as parcerias podem potencializar a conjunção de habilidades e de conhecimentos complementares ${ }^{46}$, além de discursos multidisciplinares ${ }^{47}$.

Observou-se uma aproximação das palavraschave mais frequentes com as categorias temáticas identificadas nesse estudo. As palavras-chave facilitam a recuperação de conteúdo, são fundamentais para a indexação e atuam como porta de acesso ao texto ${ }^{48}$, o que reitera a importância da seleção criteriosa delas, de modo que retratem, de fato, a temática e o conteúdo abordados. As palavras-chave e as categorias temáticas espelham as metas previstas nos planos global e nacional de enfrentamento das DCNT ${ }^{8,19}$, os Objetivos de Desenvolvimento Sustentável (ODS) e a agenda nacional de pesquisas em saúde, justificando a frequência elevada de termos como "Doenças crônicas não transmissíveis", "Fatores de risco", "Obesidade", "Tabagismo", "Atividade física", "Alimentação" e "Álcool". Salienta-se que a maior parte dos artigos se refere aos fatores de risco e de proteção para as DCNT, o que pode refletir também a consolidação da vigilância das DCNT no país. O Brasil organizou ações para estruturar e operacionalizar um sistema de vigilância específico para esse grupo de doenças, de modo a conhecer a distribuição, a magnitude e a tendência das DCNT e seus fatores de risco, apoiar as políticas públicas de prevenção e de promoção da saúde ${ }^{19}$ e, complementarmente, identificar os determinantes sociais, econômicos e ambientais, estabelecendo cadeias multicausais de determinação $0^{2,49}$. A esse respeito, cumpre mencionar a implementação de alguns inquéritos no país.

Em 2003 foi realizado o primeiro inquérito domiciliar nacional sobre comportamento de risco e morbidade referida de agravos não transmissíveis, configurando a linha de base no monitoramento dos principais fatores de risco de DCNT ${ }^{50}$. Desde 2006, implantado em todas as capitais dos 26 estados brasileiros e no Distrito Federal, o Sistema de Vigilância de Fatores de Risco e Proteção para Doenças Crônicas por Inquérito Telefônico (Vigitel) monitora a frequência e a distribuição dos principais determinantes das DCNT por inquérito telefônico ${ }^{51}$. Em 2008 a PNAD incluiu, como parte da iniciativa do GATS, informações sobre morbidade e alguns fatores de risco, no Brasil chamada: Pesquisa Especial de Tabagismo $(\mathrm{PETab})^{52}$. Em 2009, em parceria com o Instituto Brasileiro de Geografia e Estatística (IBGE) e com o apoio do Ministério da Educação (MEC), instituiu-se a PeNSE, pesquisa realizada com es- 
colares adolescentes. A pesquisa aconteceu em 2009, 2012, 2015 e 2019, tendo como objetivo subsidiar o monitoramento de fatores de risco e de proteção à saúde em escolares do Brasil ${ }^{53}$. Em 2013 e 2019, a PNS foi realizada pelo IBGE, em parceria com o MS, sendo a mais ampla pesquisa de base domiciliar do país, com o intuito de coletar informações sobre o desempenho do sistema nacional de saúde no que se refere ao acesso e ao uso dos serviços disponíveis e à continuidade dos cuidados, bem como sobre as condições de saúde da população, a vigilância de doenças crônicas não transmissíveis e os fatores de risco a elas associados ${ }^{54}$. Além desses inquéritos, a Pesquisa de Orçamento Familiar (POF) revela resultados relativos à condição nutricional da população brasileira e ao consumo alimentar dos brasileiros ${ }^{55}$.

Concernente ao financiamento das publicações analisadas, este foi proveniente de agentes públicos (agências de fomento e órgãos de governo) em mais de $90 \%$ dos casos em que o mesmo foi declarado, o que guarda relação com o fato do Estado brasileiro contar com um arcabouço legal e normativo que orienta a articulação entre os setores Saúde, Educação e Ciência e Tecnologia, bem como o desenvolvimento nacional em ciência, tecnologia e inovação em saúde. $\mathrm{O}$ fomento à realização de pesquisas e estudos na área da saúde encontra-se no cerne do SUS, materializado por meio da Lei $\mathrm{N}^{\circ} 8.080^{56}$.

O setor público também respondeu pela principal vinculação institucional dos autores que publicaram na revista. Essa informação indica que, apesar dos desafios relacionados à escassez dos recursos financeiros destinados às universidades públicas e às pesquisas, sobretudo em tempos de austeridade fiscal e subfinanciamento da ciência e tecnologia, existe uma mobilização no sentido de fomentar o cumprimento do papel social destas instituições e de seus pesquisadores. A pesquisa científica, tecnológica e de inovação constitui um elemento fundamental em todos os setores da sociedade, particularmente no âmbito e para o SUS, produzindo mudanças significativas nas vidas das pessoas, nas relações e nos processos de trabalho ${ }^{57}$. O campo da saúde responde por pouco mais de um terço da produção científica no Brasil e registram-se igualmente esforços e iniciativas bem sucedidas do $\mathrm{MS}^{58,59}$.

Este estudo identificou os pesquisadores que mais publicaram sobre DCNT na C\&SC, dos quais a maioria do sexo feminino. No Brasil, as mulheres responderam pela autoria de aproximadamente metade das publicações entre os anos de 2011 e 2015, representando um aumento em relação ao período de 1996 a 2000, cujo percentual era de $38 \%{ }^{60}$. Entretanto, as mulheres ainda têm menor proporção em relação aos homens, quanto ao recebimento de bolsas de produtividade do Conselho Nacional de Desenvolvimento Científico e Tecnológico $(\mathrm{CNPq})^{61}$.

Entre as limitações deste estudo, pode-se mencionar a utilização apenas da base de dados PubMed para recuperação automática das publicações. A escolha da base pode interferir no material recuperado, em termos quantitativos e qualitativos, em função das particularidades dos motores de pesquisa utilizados por cada base. Outrossim, deve-se levar em consideração a sensibilidade e a especificidade da estratégia de busca, que, nesse caso particular, refletiu a opção dos pesquisadores pelo enfoque dos quatro grandes grupos de DCNT e de seus fatores de risco e proteção, face a um amplo escopo de doenças crônicas.

A temática referente às DCNT foi amplamente abordada na C\&SC, que vem espelhando a magnitude, a prioridade e a transcendência da questão. Observou-se, na esfera político-institucional, a ampliação dos inquéritos nacionais - as fontes de dados sobre DCNT -, refletindo no aumento das publicações e no incremento das análises sobre fatores de risco e de proteção ao longo do tempo, com destaque para a importante liderança das instituições públicas de ensino e de pesquisa na produção desse conhecimento científico, cujas evidências, em última instância, podem subsidiar a formulação de políticas e apoiar processos de monitoramento e avaliação. A revista cumpre o seu papel divulgando evidências, promovendo o debate e ampliando o conhecimento científico sobre tema de tamanha relevância. Ademais, as DCNT emergiram como prioridade de forma coordenada nas agendas nacional e global, notadamente, a partir do plano nacional de enfrentamento, do plano de ação global e dos ODS. Diante de tal cenário, compreender a morbimortalidade, os fatores de risco e as dinâmicas de distribuição na população, monitorar esses eventos, assim como refletir sobre a gestão pública dessas questões é essencial no debate científico e também na agenda governamental. 


\section{Colaboradores}

DC Malta, AG Silva, LSM Cardoso, FMD Andrade, ACMGN Sá, EJS Prates, FTA Alves e GF Xavier Junior contribuíram e aprovaram a versão final do artigo.

\section{Agradecimentos}

Ao Conselho Nacional de Desenvolvimento Científico e Tecnológico (CNPq) pela bolsa de produtividade para DC Malta e de iniciação científica para FTA Alves. EJS Prates agradece ao Fundo Nacional de Saúde do Ministério da Saúde pela bolsa de pesquisa. AG Silva, LSM Cardoso, FMD Andrade e ACMGN Sá agradecem à Coordenação de Aperfeiçoamento de Pessoal de Nível Superior (CAPES) pelas bolsas.

E ao financiamento pelo Fundo Nacional de Saúde - FNS, Ministério da Saúde, TED 66/2018.

\section{Referências}

1. World Health Organization (WHO). Noncommunicable diseases progress monitor 2020. Geneva: WHO; 2020. [acessado $2020 \mathrm{Abr} 24$ ]. Available from: https:// www.who.int/publications-detail/ncd-progress-monitor-2020

2. Malta DC, Silva MMA, Moura L, Morais Neto OL. A implantação do Sistema de Vigilância de Doenças Crônicas Não Transmissíveis no Brasil, 2003 a 2015: alcances e desafios. Rev Bras Epidemiol 2017; 20(4):661-675.

3. Malta DC, Bernal RTI, Lima MG, Araújo SSC, Silva MMA, Freitas MIF, Barros MBA. Doenças crônicas não transmissíveis e a utilização de serviços de saúde: análise da Pesquisa Nacional de Saúde no Brasil. Rev Saude Publica 2017; 51(Supl. 1):4s.

4. Moura de AAG, Carvalho de EF, Silva NJC. Repercussão das doenças crônicas não-transmissíveis na concessão de benefícios pela previdência social. Cien Saude Colet 2007; 12(6):1661-1672.

5. World Health Organization (WHO). World Health Statistics 2015. Genebra: WHO; 2015 [acessado 2020 Abr 24]. Disponível em: https://apps.who.int/iris/ bitstream/handle/10665/170250/9789240694439_ eng.pdf?sequence $=1$ \&isAllowed $=y$

6. Pan American Health Organization (PAHO). The economic burden of Non-communicable Diseases in the Americas. Washington: PAHO; 2011. [acessado 2020 Abr 24]. Disponível em: https://www.paho.org/hq/ dmdocuments/2011/paho-policy-brief-3-En-web1. pdf

7. World Health Organization (WHO). Health 2020: a European policy framework supporting action across government and society for health and well-being. Geneva: WHO; 2013 [acessado 2020 Abr 24]. Disponível em: http://www.euro.who.int/_data/assets/ pdf_file/0006/199536/Health2020-Short.pdf

8. World Health Organization (WHO). Global Action Plan for the Prevention and Control of NCDs 20132020. Geneva: WHO; 2013 [acessado 2020 Abr 24]. Disponível em: https://apps.who.int/iris/bitstream/ handle/10665/94384/9789241506236_eng.pdf;jsessionid $=$ CC05322BD984F039B84D717FF29E96D8? sequence $=1$

9. Paes-Sousa R, Schramm JMA, Mendes LVP. Austeridade fiscal e o setor saúde: o preço do ajuste. Cien Saude Colet 2019; 24(12):4375-4384.

10. Malta DC, Duncan BB, Barros MBA, Katikireddi SV, Souza FM, Silva AG, Machado DB, Barreto ML. Medidas de austeridade fiscal comprometem metas de controle de doenças não transmissíveis no Brasil. Cien Saude Colet 2018; 23(10):3115-3122.

11. Schmidt MI, Duncan BB, Azevedo e Silva G, Menezes AM, Monteiro CA, Barreto SM, Chor D, Menezes PR. Chronic non-communicable diseases in Brazil: burden and current challenges. Lancet 2011; 377(9781):1949-1961.

12. Marmot M, Bell R. Social determinants and noncommunicable diseases: time for integrated action. BMJ 2019; 365(Supl. 1):kl251. 
13. Stringhini $S$, Carmeli C, Jokela $M$, Avendaño $M$, Muennig P, Guida F, Ricceri F, d'Errico A, Barros H, Bochud M, Chadeau-Hyam M, Clavel-Chapelon F, Costa G, Delpierre C, Fraga S, Goldberg M, Giles GG, Krogh V, Kelly-Irving M, Layte R, Lasserre AM, Marmot MG, Preisig M, Shipley MJ, Vollenweider P, Zins M, Kawachi I, Steptoe A, Mackenbach JP, Vineis P, Kivimäki M, LIFEPATH consortium. Socioeconomic status and the $25 \times 25$ risk factors as determinants of premature mortality: a multicohort study and meta -analysis of 1.7 million men and women. Lancet 2017; 389(10075):1229-1237.

14. Pearce N, Ebrahim S, McKee M, Lamptey P, Barreto ML, Matheson D, Walls H, Foliaki S, Miranda JJ, Chimeddamba O, Garcia-Marcos L, Haines A, Vineis P. Global prevention and control of NCDs: Limitations of the standard approach. J Public Health Policy 2015; 36(4):408-425.

15. Malta DC, Bernal RT, de Souza MF, Szarcwald CL, Lima MG, Barros MB. Social inequalities in the prevalence of self-reported chronic non-communicable diseases in Brazil: national health survey 2013. Int J Equity Health 2016; 15:153.

16. Vošner HB, Kokol P, Bobek S, Železnik D, Završnik J. A bibliometric retrospective of the Journal Computers in Human Behavior (1991-2015). Comput Human Behav 2016; 65:46-58.

17. Araújo CA. Bibliometria: evolução histórica e questões atuais. Em Questão 2006; 12(1):11-32.

18. Brasil. Ministério da Saúde (MS). Plano de ações estratégicas para o enfrentamento das doenças crônicas não transmissíveis (DCNT) no Brasil 2011-2022. Brasília: MS; 2011 [acessado 2020 Abr 24]. Disponível em: https://bvsms.saude.gov.br/bvs/publicacoes/plano_acoes_enfrent_dent_2011.pdf

19. Lunardi MS, Castro JMFC, Monat AS. Visualização dos resultados do Yahoo em nuvens de texto: uma aplicação construída a partir de web services. InfoDesign - Revista Brasileira de Design da Informação 2008; 5(1):21-35.

20. World Health Organization (WHO). Prevention and control of noncommunicable diseases. Genebra: WHO; 2000 [acessado 2020 Abr 23]. Disponível em: https://www.paho.org/bra/index. php?option $=$ com_docman\&view $=$ download $\&$ alias $=1178$-resolucao-53-17-prevencao-e-controledoencas-cronicas-nao-transmissiveis-8\&category slug=doencas-nao-transmissiveis-948\&Itemid $=965$

21. Malta DC, Cezário AC, Moura L, Morais Neto OL, Silva Junior JB. A construção da vigilância e prevenção das doenças crônicas não transmissíveis no contexto do Sistema Único de Saúde. Epidemiol Serv Saúde 2006; 15(3):47-65.

22. Brasil. Ministério da Saúde (MS). A vigilância, o controle e a prevenção das doenças crônicas não transmissiveis: DCNT no contexto do Sistema Único de Saúde brasileiro / Brasil. Brasília: MS; 2005 [acessado 2020 Abr 24]. Disponível em: https://www.saude.gov.br/ images/pdf/2015/fevereiro/23/2.\%20DCNT\%20 no\%20contexto\%20do\%20SUS\%202005.pdf

23. Almeida MF, Barata RB, Montero CV, Silva ZP. Prevalência de doenças crônicas auto-referidas e utilização de serviços de saúde, PNAD/1998, Brasil. Cien Saude Colet 2002; 7(4):743-756.
24. Leite IC, Schramm JMA, Gadelha AMJ, Valente JG Campos MR, Portela MC, Hokerberg YHM, Oliveira AF, Cavalini LT, Ferreira VMB, Bittencourt SA. Comparação das informações sobre as prevalências de doenças crônicas obtidas pelo suplemento saúde da PNAD/98 e as estimadas pelo estudo Carga de Doença no Brasil. Cien Saude Colet 2002; 7(4):733-741.

25. Iwakami BK, Sonoê S. Comparação de informações sobre saúde das populações brasileira e norte-americana baseada em dados da PNAD/98 e NHIS/96. Cien Saude Colet 2002; 7(4):841-867.

26. Lessa I. Editorial. Cien Saude Colet 2004; 9(4):828831.

27. Lessa I. Doenças crônicas não-transmissíveis no Brasil: um desafio para a complexa tarefa da vigilância. Cien Saude Colet 2004; 9(4):931-943.

28. Achutti A, Azambuja MIR. Doenças crônicas nãotransmissíveis no Brasil: repercussões do modelo de atenção à saúde sobre a seguridade social. Cien Saude Colet 2004; 9(4):833-840.

29. Loureiro S. Modelos assistenciais podem diminuir o impacto das DCNT na seguridade social? Cien Saude Colet 2004; 9(4):843:844.

30. Lotufo PA. Por que não vivemos uma epidemia de doenças crônicas: o exemplo das doenças cardiovasculares?. Cien Saude Colet 2004; 9(4):844-847.

31. Teixeira CF. Transição epidemiológica, modelo de atenção à saúde e previdência social no Brasil: problematizando tendências e opções políticas. Cien Saude Colet 2004; 9(4):841-843.

32. Almeida-Filho N. Modelos de determinação social das doenças crônicas não-transmissíveis. Cien Saude Colet 2004; 9(4):865-884.

33. Lima-Costa MF. Estilos de vida e uso de serviços preventivos de saúde entre adultos filiados ou não a plano privado de saúde (inquérito de saúde de Belo Horizonte). Cien Saude Colet 2004; 9(4):857-864.

34. Laurenti R, Mello Jorge MHP, Gotlieb SLD. A confiabilidade dos dados de mortalidade e morbidade por doenças crônicas não-transmissíveis. Cien Saude Colet 2004; 9(4):909-920.

35. Toscano CM. As campanhas nacionais para detecção das doenças crônicas não-transmissíveis: diabetes e hipertensão arterial. Cien Saude Colet 2004; 9(4):885895.

36. Franco LJ, Campos GP, Machado CA. Entrevista: O enfoque das políticas do SUS para promoção da saúde e prevenção das DCNT: do passado ao futuro. Cien Saude Colet 2004; 9(4):945-956.

37. Coordenação Geral de Doenças e Agravos Não-Transmissíveis. Síntese da oficina de vigilância em doenças crônicas não-transmissíveis. Cien Saude Colet 2004; 9(4):957-962.

38. Malta DC, Sardinha LMV, Mendes I, Barreto SM, Giatti L, Castro IRR, Moura L, Dias AJR, Crespo C. Prevalência de fatores de risco e proteção de doenças crônicas não transmissíveis em adolescentes: resultados da Pesquisa Nacional de Saúde do Escolar (PeNSE), Brasil, 2009. Cien Saude Colet 2010; 15(Supl. 2):3009-3019.

39. Levy RB, Castro IRR, Cardoso LO, Tavares LF, Sardinha LMV, Gomes FS, Costa AWN. Consumo e comportamento alimentar entre adolescentes brasileiros: Pesquisa Nacional de Saúde do Escolar (PeNSE), 2009. Cien Saude Colet 2010; 15(Supl. 2):3085-3097. 
40. Castro IRR, Levy RB, Cardoso LO, Passos MD, Sardinha LMV, Tavares LF, Dutra SP, Martins A. Imagem corporal, estado nutricional e comportamento com relação ao peso entre adolescentes brasileiros. Cien Saude Colet 2010; 15(Supl. 2):3099-3108.

41. Hallal PC, Knuth AG, Cruz DKA, Mendes MI, Malta DC. Prática de atividade física em adolescentes brasileiros. Cien Saude Colet 2010; 15(Supl. 2):3035-3042.

42. Sánchez FG, Terry JGP, Goire CT, Prevots MO, Castillo MC. Análisis de algunos indicadores bibliométricos de la Revista Información Científica de la Universidad Médica de Guantánamo. Rev Inf Cient 2018; 97(6):1088-1099.

43. Conner N, Provedel A, Maciel ELN. Ciência \& Saúde Coletiva: análise da produção científica e redes colaborativas de pesquisa. Cien Saude Colet 2017; 22(3):987-996.

44. Sweileh WM, Al-Jabi SW, AbuTaha AS, Zyoud SH, Anayah FMA, Sawalha AF. Bibliometric analysis of worldwide scientific literature in mobile - health: 2006-2016. BMC Med Inform Decis Mak 2017; 17(1):72.

45. Njelesani J, Stevens M, Cleaver S, Mwambwa L, Nixon S. International research partnerships in occupational therapy: A Canadian-Zambian case study. Occup Ther In 2013; 20(2):75-84.

46. Luz MT. Complexidade do campo da Saúde Coletiva: multidisciplinaridade, interdisciplinaridade, e transdisciplinaridade de saberes e práticas - análise sócio -histórica de uma trajetória paradigmática. Saude Soc 2009; 18(2):304-311.

47. Garcia DCF, Gattaz CC, Gattaz NC. A Relevância do Título, do Resumo e de Palavras-chave para a Escrita de Artigos Científicos. Rev Adm Contemp 2019; 23(3):1-9.

48. Bonita R, Magnusson R, Bovet P, Zhao D, Malta DC Geneau R, Il S, Thankappan KR, McKee M, Hospedales J, Courten M, Capewell S, Beaglehole R, Lancet NCD Action Group. Country actions to meet UN commitments on non-communicable diseases: a stepwise approach. Lancet 2013; 381(9866):575-584.

49. Instituto Nacional de Câncer (INCA). Inquérito domiciliar sobre comportamentos de risco e morbidade referida de doenças e agravos não transmissiveis: Brasil, 15 capitais de Distrito Federal, 2002-2003. Rio de Janeiro: INCA; 2004 [acessado 2020 Abr 24]. Disponível em: https://bvsms.saude.gov.br/bvs/publicacoes/ inquerito_domiciliar_comportamentos_risco_doencas_transmissiveis.pdf

50. Brasil. Ministério da Saúde (MS). Vigitel Brasil 2006: vigilância de fatores de risco e proteção para doenças crônicas por inquérito telefônico. Brasília: MS; 2007 [acessado 2020 Abr 24]. Disponível em: https://bvsms.saude.gov.br/bvs/publicacoes/vigitel_brasil_2006. pdf

51. Instituto Brasileiro de Geografia e Estatística (IBGE). Pesquisa Nacional por Amostra de Domicílios: tabagismo 2008. Rio de Janeiro: IBGE; 2009 [acessado 2020 Abr 24]. Disponível em: https://biblioteca.ibge.gov. br/visualizacao/livros/liv42980.pdf

52. Brasil. Ministério da Saúde (MS). Pesquisa Nacional de Saúde do Escolar (PeNSE):o que é, para que serve, temas [página na Internet]. [acessado $2020 \mathrm{Abr} 24$ ]. Disponível em: https://saude.gov.br/saude-de-a-z/ pense
53. Instituto Brasileiro de Geografia e Estatística (IBGE). Pesquisa Nacional de Saúde - PNS [página na Internet]. [acessado 2020 Abr 24]. Disponível em: https://www.ibge.gov.br/estatisticas/sociais/justica -e-seguranca/9160-pesquisa-nacional-de-saude.ht$\mathrm{ml}$ ?=\&amp; $\mathrm{t}=\mathrm{o}$-que-e

54. Instituto Brasileiro de Geografia e Estatística (IBGE) Pesquisa de Orçamentos Familiares - POF [página na Internet]. [acessado 2020 Abr 24]. Disponível em: https://www.ibge.gov.br/estatisticas/sociais/trabalho/ 9050-pesquisa-de-orcamentos-familiares.html?edi$\mathrm{cao}=9060 \& \mathrm{t}=$ sobre

55. Brasil. Lei 8080 de 19 de Setembro de 1990. Dispõe sobre as condições para a promoção, proteção e recuperação da saúde, a organização e o funcionamento dos serviços correspondentes e dá outras providências. Diário Oficial da União 1990; 20 set.

56. Tenório M, Mello GA, Viana ALD. Políticas de fomento à ciência, tecnologia e inovação em saúde no Brasil e o lugar da pesquisa clínica. Cien Saude Colet 2017; 22(5):1441-1454

57. Departamento de Ciência e Tecnologia, Secretaria de Ciência, Tecnologia e Insumos Estratégicos, Ministério da Saúde. Pesquisa em saúde no Brasil. Rev Saude Publica 2008; 42(4):773-775.

58. Santos LMP, Souza LEPF, Serruya SJ, Guimarães RFN. O papel da pesquisa na consolidação do Sistema Único de Saúde (SUS). Cad Saude Publica 2010; 26(9):1666-1667.

59. Goldbaum M, Serruya SJ. O Ministério da Saúde e a política de ciência, tecnologia e inovação em saúde. Cad Saude Publica 2006; 22(3):470-471.

60. Carvalho MS, Coeli CM, Lima LD. Mulheres no mundo da ciência e da publicação científica. Cad Saude Publica 2018; 34(3):e00025018.

61. Valentova JV, Otta E, Silva ML, McElligott AG. Underrepresentation of women in the senior levels of Brazilian science. PeerJ 2017; 5:e4000.
Artigo apresentado em 27/05/2020

Aprovado em 28/05/2020

Versão final apresentada em 30/05/2020 
